\title{
ELENCO DEI COLLABORATORI
}

Alfredo Antonini, Professore associato di Diritto della navigazione, Università di Modena

Silvio BuSTI, Professore associato di Diritto della navigazione, Università di Trento

DOMEniCo MALTESE, Procuratore Generale della Repubblica, Corte d'Appello di Trieste

Elena OrSetTa QuerCI, Dottore in Giurisprudenza, Università di Roma

Maria PIERA RIzzO, Ricercatore di Diritto internazionale Università di Reggio Calabria

JON M. VAN DYKe, Professor of Law, University of Hawaii at Manoa, Honolulu 